\title{
Plantas protegidas ligadas a los medios húmedos en España: presiones y amenazas
}

DOI: $10.15366 / \mathrm{cv} 2019.23 .003$

\section{Introducción}

El pasado 28 de septiembre de 2018 se entregó al Centro de Estudio y Experimentación de Obras Públicas (CEDEX) el Informe Final del proyecto realizado por la SEBiCoP* titulado "Análisis de la distribución actual, estado de conservación y requerimientos ecológicos de 38 especies de plantas protegidas ligadas al agua". Este contrato, impulsado desde el entonces Ministerio de Agricultura, Pesca, Alimentación y Medio Ambiente (MAPAMA), en colaboración con el CEDEX, tenía como objetivo obtener información de 38 especies seleccionadas por el MAPAMA (21 angiospermas, 11 pteridófitos y 6 briófitos), relacionadas con los hábitats húmedos en sentido amplio.

\begin{tabular}{|l|l|}
\hline Apium bermejoi & Marsilea batardae \\
\hline Apium repens & Marsilea quadrifolia \\
\hline Bruchia vogesiaca & Marsilea strigosa \\
\hline Caropsis verticillato-inundata & Micropyropsis tuberosa \\
\hline Centaurium somedanum & Narcissus cyclamineus \\
\hline Christella dentata & Narcissus longispathus \\
\hline Coronopus navasii & Narcissus nevadensis \\
\hline Culcita macrocarpa & Nuphar luteum subsp. pumilum \\
\hline Diplazium caudatum & Pilularia minuta \\
\hline Echinodium spinosum & Pinguicula nevadensis \\
\hline Eryngiium viviparum & Pteris incompleta \\
\hline Galium viridiflorum & Puccinellia fasciculata subsp. pungens \\
\hline Hamatocaulis vernicosus & Riella helicophylla \\
\hline Hymenophyllum wilsonii & Soldanella villosa \\
\hline Jungermania handelii & Sphagnum pylaisii \\
\hline Kosteletzkya pentacarpa & Tephroseris elodes \\
\hline Leontodon microcephalus & Vandenboschia speciosa \\
\hline Luronium natans & Veronica micrantha \\
\hline Lythrum flexuosum & Woodwardia radicans \\
\hline
\end{tabular}

Tabla 1. Relación de taxones estudiados en el proyecto

De cada especie, en función de su abundancia, se seleccionaron entre 1 y 12 cuadrículas UTM de $1 \times 1 \mathrm{~km}$ como Elementos Visitables (EV), en los que había constancia reciente de la presencia del taxón. En cada uno de estos EV se prospectó si persistía, y en este caso, se censó y cartografió con el máximo detalle posible la o las poblaciones, se tomaron datos biológicos y poblacionales de la planta, datos físicos y caracterización del hábitat, régimen hidrológico y morfología de la masa de agua a la que se supone adscrita la planta, indicadores físico-químicos del agua presente en el momento de muestreo, así como los posibles contaminantes (amonio, nitratos, fosfatos). La información se recogió según tres tipos de hábitat: Corrientes de agua, Humedales y Ambientes higrófilos. Como Corrientes de agua se entiende cualquier corriente superficial, intermitente o no. Como Humedal se consideró cualquier embalsamiento de agua, intermitente o no. Como Ambiente higrófilo se incorporaron los suelos encharcados, taludes rezumantes, turberas, criptohumedales, suelo hidromorfo, punto de descarga y, en general, aquellos ambientes que no encajen en los dos primeros grupos.
Los factores de amenaza se anotaron en campo siguiendo "The list of pressures/threat" versión del 13 de octubre de 2017 (EEA, 2019), una lista estándar de presiones y amenazas elaborada para identificar los factores de amenaza para los taxones y cumplir así de manera homogénea con las obligaciones del artículo 17 de la Directiva de Hábitats: informar sobre el estado de conservación de especies y hábitats. Con esta información cada experto responsable de un taxón elaboró un informe, en el que incluyeron las directrices de gestión que estimó necesarias para asegurar la supervivencia y buena gestión del hábitat de las poblaciones de las especies estudiadas.

Se han recogido aproximadamente 800 poblaciones, localizadas en 352 cuadrículas UTM 1x1 (215 cUTM 10×10 km) distribuidas por todo el territorio español (Figura 1). De cada una en campo se anotaron, además del resto de datos que no se tienen en consideración aquí, las presiones actuales y amenazas futuras que le afectan. En este artículo analizamos las principales presiones y amenazas detectadas para las 38 especies de este proyecto mediante el estudio de la cantidad de veces que han sido anotadas por los equipos de campo. Con los datos reunidos hemos hecho unas gráficas sencillas en las que figuran el número total de veces que aparece cada presión o amenaza y su porcentaje relativo de frecuencia.

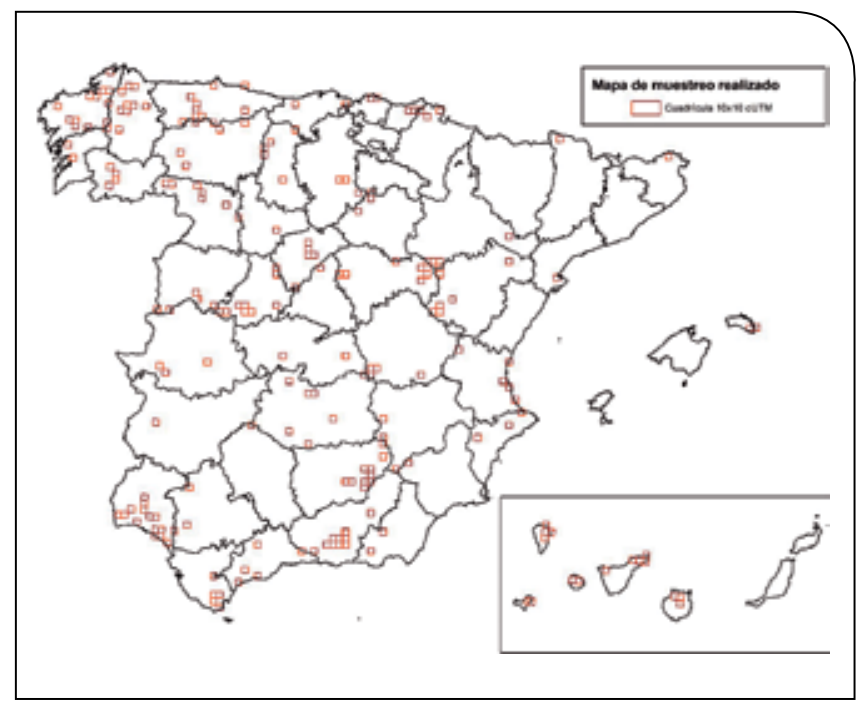

Figura 1. Mapa de distribución de las cuadrículas muestreadas (302 cUTM de 1x1 km en la Península y Baleares, 199 cUTM de 10x10 km; 50 cUTM 1x1 $\mathrm{km}$ en las Islas Canarias, 16 cUTM 10×10 km).

\section{Resultados}

Lo primero que destaca al analizar los datos es que la inmensa mayoría de los factores de riesgo para las especies estudiadas derivan de las actividades humanas, con más del $90 \%$ de las anotadas, mientras que no llegan ni al 10\% los derivados de procesos naturales y eventos geológicos o catástrofes. Las principales presiones detectadas son, con gran diferencia, la 
ganadería y la agricultura y en un segundo escalón le siguen el manejo forestal, la contaminación y el cambio climático (Figura 2). En cuanto a las Amenazas potenciales, la principal es el Cambio climático, con un $41 \%$, y le sigue la Agricultura con un $12 \%$.

Si analizamos por ambientes (Tabla 2), lo más destacable es el predominio de las presiones derivadas de la agricultura y la ganadería para todos los tipos de hábitat. En cambio, cada uno de ellos está afectado de manera secundaria por presiones diferentes: las Corrientes de agua por el manejo forestal y los sistemas de transporte, los Humedales por el urbanismo y el cambio del régimen de aguas, y los Ambientes higrófilos por la invasión de especies alóctonas y los eventos geoló- gicos y catástrofes.Entre los factores de amenaza a futuro que los equipos de campo han anotado con más frecuencia (Tabla 3) figura el cambio climático para los tres hábitats, y también coinciden en que el segundo factor de amenaza es la Agricultura (bajo este epígrafe se reúne tanto la agricultura como la ganadería).

En conclusión, el elevado número de poblaciones estudiado y su distribución geográfica permiten ofrecer una imagen de los principales factores de amenaza sobre el conjunto de los hábitats ligados a la humedad edáfica de España, y concluir que las actividades humanas son su principal riesgo, especialmente la agricultura y la ganadería como presión actual y el cambio climático como amenaza futura.

\section{(A) Presiones}

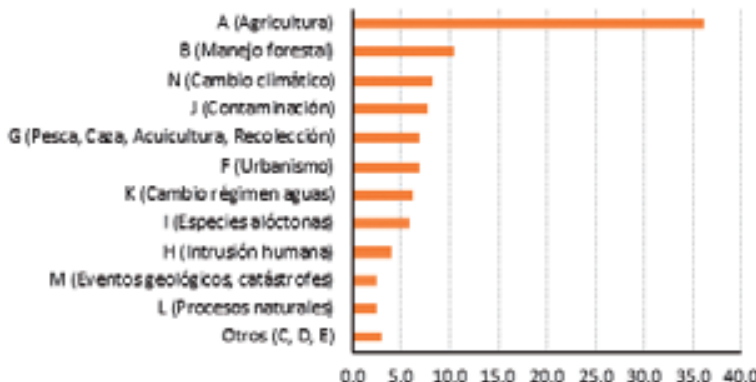

\section{(B) Amenazas}

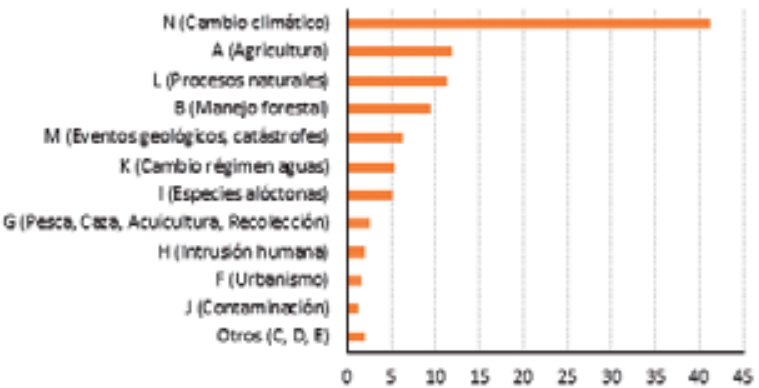

Figura 2.

Frecuencia (\%) con la que se han identificado los distintos tipos de (A) Presiones actuales y (B) Amenazas potenciales [A: Agricultura y ganadería; B: manejo forestal; C: extracción de recursos: D: producción de energía; $\mathrm{E}$ : sistemas de transporte; $\mathrm{F}$ : urbanismo: G: pesca, caza, acuicultura, recolección: $\mathrm{H}$ : intrusión humana; I: especies alóctonas; J: contaminación; K: cambio régimen aguas; L: procesos naturales; M: eventos geológicos, catástrofes; N: cambio climático).

\begin{tabular}{|c|c|c|c|c|c|c|c|c|c|c|c|c|c|c|c|}
\hline \multicolumn{16}{|c|}{ PRESIONES ACTUALES } \\
\hline \multicolumn{2}{|c|}{$\begin{array}{l}\text { TIPO DE MASA } \\
\text { DE AGUA }\end{array}$} & \multirow{2}{*}{ 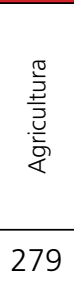 } & \multirow{2}{*}{ 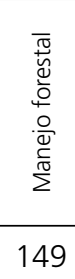 } & \multirow{2}{*}{ 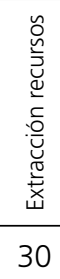 } & \multirow{2}{*}{ 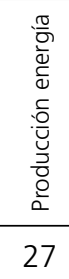 } & \multirow{2}{*}{ 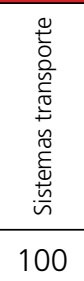 } & \multirow{2}{*}{ 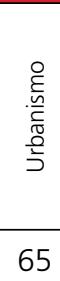 } & \multirow{2}{*}{ 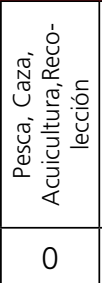 } & \multirow{2}{*}{ 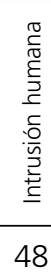 } & \multirow{2}{*}{ 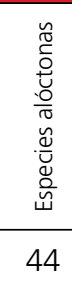 } & \multirow{2}{*}{ 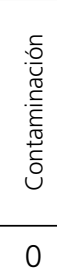 } & \multirow{2}{*}{ 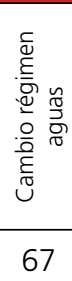 } & \multirow{2}{*}{ 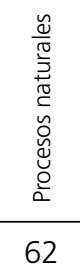 } & \multirow{2}{*}{ 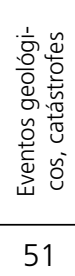 } & \multirow{2}{*}{ 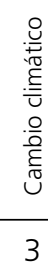 } \\
\hline \multirow{2}{*}{ rientes de agua } & $\mathrm{N}^{\circ}$ & & & & & & & & & & & & & & \\
\hline & $\%$ & 30,2 & 16,1 & 3,2 & 2,9 & 10,8 & 7 & 0 & 5,2 & 4,8 & 0 & 7,2 & 6,7 & 5,5 & 0,3 \\
\hline \multirow{2}{*}{ Humedales } & $\mathrm{N}^{\circ}$ & 253 & 21 & 11 & 4 & 33 & 46 & 1 & 11 & 8 & 13 & 47 & 36 & 4 & 0 \\
\hline & $\%$ & 51,8 & 4,3 & 2,3 & 0,8 & 6,8 & 9,4 & 0,2 & 2,3 & 1,6 & 2,7 & 9,6 & 7,4 & 0,8 & 0 \\
\hline \multirow{2}{*}{$\begin{array}{l}\text { Ambiente } \\
\text { higrófilo }\end{array}$} & $\mathrm{N}^{\circ}$ & 79 & 17 & 3 & 13 & 15 & 10 & 6 & 14 & 52 & 10 & 5 & 38 & 53 & 19 \\
\hline & $\%$ & 23,7 & 5,1 & 0,9 & 3,9 & 4,5 & 3 & 1,8 & 4,2 & 15,6 & 3 & 1,5 & 11,4 & 15,9 & 5,7 \\
\hline
\end{tabular}

Tabla 2. Frecuencia de las principales presiones actuales.

\begin{tabular}{|c|c|c|c|c|c|c|c|c|c|c|c|c|c|c|c|}
\hline \multicolumn{16}{|c|}{ AMENAZAS POTENCIALES } \\
\hline \multicolumn{2}{|c|}{$\begin{array}{c}\text { TIPO DE MASA } \\
\text { DE AGUA }\end{array}$} & $\frac{\stackrel{0}{3}}{\frac{ \pm}{3}}$ & 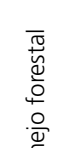 & 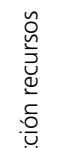 & $\begin{array}{l}\frac{0}{0} \\
\frac{0}{2} \\
\frac{c}{d} \\
: \frac{0}{U} \\
: \frac{0}{U}\end{array}$ & 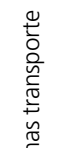 & 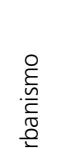 & 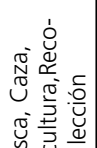 & 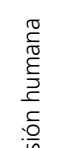 & 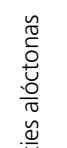 & 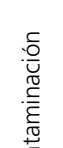 & 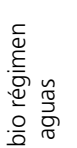 & 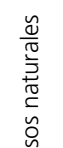 & 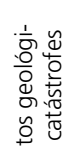 & 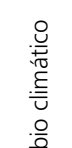 \\
\hline \multirow{2}{*}{ Corrientes de agua } & $N^{\circ}$ & 106 & 103 & 12 & 12 & 19 & 19 & 0 & 10 & 29 & 7 & 74 & 77 & 32 & 270 \\
\hline & $\%$ & 13,8 & 13,4 & 1,6 & 1,6 & 2,5 & 2,5 & 0 & 1,3 & 3,8 & 0,9 & 9,6 & 10 & 4,2 & 35,1 \\
\hline \multirow{2}{*}{ Humedales } & $\mathrm{N}^{\circ}$ & 121 & 16 & 4 & 11 & 6 & 3 & 21 & 8 & 26 & 1 & 22 & 22 & 15 & 144 \\
\hline & $\%$ & 28,8 & 3,8 & 1 & 2,6 & 1,4 & 0,7 & 5 & 1,9 & 6,2 & 0,2 & 5,2 & 5,2 & 3,6 & 34,3 \\
\hline \multirow{2}{*}{$\begin{array}{l}\text { Ambiente } \\
\text { higrófilo }\end{array}$} & $\mathrm{N}^{\circ}$ & 41 & 17 & 0 & 3 & 3 & 6 & 4 & 32 & 3 & 4 & 24 & 20 & 82 & 128 \\
\hline & $\%$ & 11,2 & 4,6 & 0 & 0,8 & 0,8 & 1,6 & 1,1 & 8,7 & 0,8 & 1,1 & 6,5 & 5,4 & 22,3 & 34,9 \\
\hline
\end{tabular}

Tabla 3. Frecuencia de las principales amenazas potenciales 


\section{Agradecimientos}

A todos los que han participado y ayudado a llevar a buen puerto este proyecto, sobre todo en el trabajo de campo: técnicos de espacios naturales, agentes medioambientales, especialistas y todos los que han colaborado de alguna manera.
* Este trabajo se ha realizado con un amplio equipo de expertos botánicos socios de la SEBiCOP, coordinados por todo el Estado, y cuyos responsables fueron Jaime Fagúndez (UDC), José Antonio Fernández Prieto (UNIOVI), Joseba Garmendia Altuna (Soc. Ci. Aranzadi), Daniel Goñi Martínez (Larre Consultores), Llorenç Sáez Gonyalons y Moisès Guardiola (UAB), Ricardo Garilleti Álvarez y Belén Albertos (UV), Llorenç Gil Vives (UIB), Carmen Acedo Casado (ULE), José Ángel Sánchez Agudo (USAL), Felipe Martínez García (UPM), Leopoldo Medina Sancho (UCM), Carlos Salazar Mendías (UJA), Julio Peñas de Giles (UGR), Laura Sánchez Villegas, Isabel Saro Hernández (ULPGC) y Patxi Heras Pérez (Museo Ci. Nat. Álava).

\section{Bibliografía} - EEA, European Environment Agency (2019). List
of pressures and threats and conservation measures with specific guidance on the use of distinct pressure and measure codes under Article 17 of
the Habitats Directive. http://cdr.eionet.europa.eu/

help/habitats art17

FELIPE MARTÍNEZ GARCÍA ${ }^{1}$, JORGE MARTÍNEZ RODRÍGUEZ², CARLOS SALAZAR MENDÍAS ${ }^{3}$

1. Departamento de Sistemas y Recursos Naturales, Universidad Politécnica de Madrid. E-mail: felipe.martinez@upm.es. 2. E-mail: jorgemartinez.sefa@gmail.com. 3. Dpto. Biología Animal, Biología Vegetal y Ecología, Universidad de Jaén. E-mail: csalazar@ujaen.es

\section{Una historia de dos bosques: el ocaso de la vegetación subtropical Afro-Macaronésica}

Este artículo es una versión del artículo galardonado en la segunda edición de los Premios César Gómez Campo en la categoría mejor trabajo científico

- Mairal, M., J. Caujapé-Castells, L. Pellissier, R. Jaén-Molina, N. Álvarez, M. Heuertz \& I. Sanmartín (2018). A tale of two forests: ongoing aridification drives population decline and genetic diversity loss at continental scale in Afro-Macaronesian evergreen-forest archipelago endemics. Annals of Botany 122: 1005-1017.

Cuesta imaginar que hace tan solo siete millones de años, cuando el Mioceno estaba llegando a su fin, el norte de África era un territorio húmedo, donde se extendía una vegetación de carácter subtropical. Sin embargo, un intenso periodo de actividad tectónica estaba a punto de comenzar: la Placa Africana se estaba elevando en su parte este, en un proceso que iba a formar los actuales arcos montañosos en torno al Gran Valle del Rift (Figura 1). Este levantamiento topográfico creó una gran sombra de lluvia orográfica, que dio lugar a los primeros signos de aridificación en el centro y oeste del continente. Posteriormente, esto se vio potenciado con la llegada del Plioceno Medio (hace 3.5 millones de años), periodo que iba a desencadenar un gran calentamiento global, afectando profundamente a las floras al sudoeste del Paleártico.

En su conjunto, todos estos cambios provocaron una enorme aridificación, lenta y gradual, que obligó a que muchas especies migraran hacia hábitats similares a los de sus nichos climáticos, fragmentaran sus poblaciones o se extinguieran. El resultado fue la extirpación de numerosas especies en el norte de África y la formación del desierto del Sáhara (Axelrod \& Raven 1978). Pero muchas de esas especies no se extinguieron totalmente, sino que algunos de sus representantes migraron a refugios climáticos en los bordes del continente e islas adyacentes: áreas con un carácter más húmedo, debido a su topografía (p. ej. cordillera del Atlas y macizo Etíope) o a la influencia de un suave clima oceánico (p. ej. Islas Canarias, Cabo Verde y Archipiélago de Socotra; Figura 1).

\section{DOI: $10.15366 / \mathrm{cv} 2019.23 .004$}

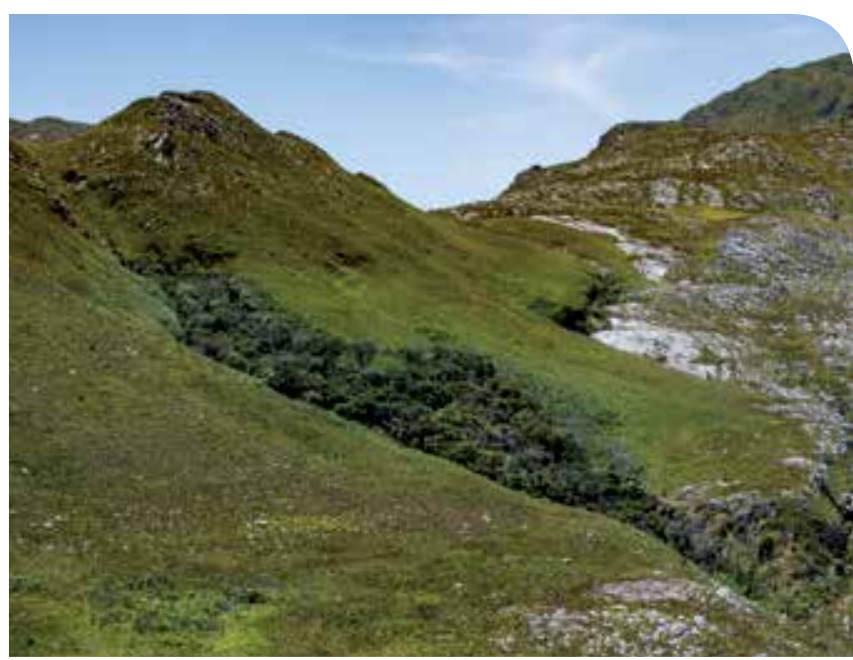

Figura 1: Retazo de bosque Afromontano. (Foto Mario Mairal)

De esta manera, linajes hermanos quedaron aislados en ambos extremos del continente africano en un fenómeno conocido como vicarianza ecológica. A su vez, y debido a que la gran aridificación de África fue gradual, los linajes no migraron súbitamente, sino que siguieron una secuencia temporal que se ajustó a su nicho climático. Es decir, los taxones de carácter subtropical, más sensibles a la aridez, fueron los primeros en desplazarse, mientras que los taxones xerofíticos, con mayor tolerancia a ambientes secos, resistieron durante más tiempo (Mairal et al. 2017a). Así, muchas de las especies que antes estaban ampliamente distribuidas se convirtieron en especies relictas - especies raras con distribución reducida- y en paleo-endemismos - especies relictas que han sufrido una amplia extinción en su rango de distribución original-. Estos paleo-endemismos son los únicos supervivientes de ramas del árbol de la vida muy "podadas" (linajes reduci- 